\title{
SOME OBSERVATIONS ON PRODUCTS OF CHARACTERS OF FINITE CLASSICAL GROUPS
}

\author{
GERHARD HISS AND FRANK LÜBECK
}

\begin{abstract}
This is an extended version of the talk given by the first author at the conference. We report on the outcome of some experiments with the decomposition of products of (generalized) characters of some classical groups. In particular some results of the PhD-thesis of Dirk Mattig on the products of unipotent characters of general linear groups are presented and commented. In order to obtain similar patterns for other classical groups it seems necessary to replace unipotent characters by unipotent almost characters.
\end{abstract}

\section{INTRODUCTION}

The unipotent characters of the general linear group $\mathrm{GL}_{n}(q)$ are parametrized by partitions of $n$, independently of $q$. Let us fix $n$ and consider three partitions $\lambda, \mu$, and $\nu$ of $n$. For each prime power $q$, there are three associated unipotent characters $\chi_{\lambda}^{q}, \chi_{\mu}^{q}$, and $\chi_{\nu}^{q}$ of $\mathrm{GL}_{n}(q)$. We may consider the multiplicity of $\chi_{\lambda}^{q}$ in the product $\chi_{\mu}^{q} \cdot \chi_{\nu}^{q}$, as a function in $q$. Following Mattig, we show that this multiplicity function is a polynomial function over the rationals. Using CHEVIE, Mattig has computed the corresponding polynomials for all $1 \leq n \leq 8$ (and all relevant triples of partitions). He found that in fact all these polynomials have non-negative integral coefficients.

After a discussion of Mattig's results, we present similar computations for the general unitary groups $\mathrm{GU}_{n}(q)$. We also consider the problem of extending our observations to other series of classical groups. We suggest to replace unipotent characters by unipotent almost characters.

Finally, we sketch some special results. For instance, we show that the constant coefficient of the polynomial expressing the multiplicity of $\chi_{\nu}^{q}$ in the square of the Steinberg character, is equal to the degree of the irreducible character of the symmetric group $S_{n}$ corresponding to $\nu$. This fact has been observed by Lux and Malle on an example presented in the talk of the first author.

Let us close this introduction by introducing two notational conventions. Let $X$ be a subset of $\mathbb{C}$ and $f: X \rightarrow \mathbb{C}, x \mapsto f(x)$ a function, and let $S \subseteq \mathbb{C}$. We say that $f$ is a polynomial in $x$ over $S$, if there is a polynomial $p \in \mathbb{C}[X]$, with coefficients in $S$, such that $f(x)=p(x)$ for all $x \in X$.

If $G$ is a finite group and if $\varphi, \psi$ are class functions on $G$, we write

$$
(\varphi, \psi)_{G}=\frac{1}{|G|} \sum_{g \in G} \varphi(g) \psi\left(g^{-1}\right)
$$

for the usual scalar product on the set of class functions of $G$. 


\section{TENSOR PRODUCT POLYNOMIALS}

Let $n$ be a positive integer, $q$ a prime power and let $G$ denote one of the groups $\mathrm{GL}_{n}(q)$ or $\mathrm{GU}_{n}(q)$. The unipotent characters of $G$ form a distinguished subset of the set of absolutely irreducible ordinary characters of $G$ (over the complex numbers).

In case $G=\mathrm{GL}_{n}(q)$ the unipotent characters of $G$ are the irreducible constituents of the permutation character $1_{B}{ }^{G}$, where $B$ denotes the Borel subgroup of upper triangular matrices of $G$. There is no elementary way to introduce the unipotent characters in case $G=\mathrm{GU}_{n}(q)$. Here, they are constructed as characters on $\ell$ adic cohomology groups on Deligne-Lusztig varieties on which $G$ acts (see, e.g., [2, Chapter 12]).

There is, however, a common parametrization of the unipotent characters in both cases. Namely, there is a bijection between the unipotent characters of $G$ and the partitons of $n$. In particular, the unipotent characters are parametrized independently of $q$. Let us write $\chi_{\lambda}^{q}$ for the unipotent character of $G$ labelled by the partiton $\lambda$ of $n$. In this parametrization $\chi_{(n)}^{q}$ denotes the trivial character $1_{G}$ and $\chi_{\left(1^{n}\right)}^{q}$ the Steinberg character $\mathrm{St}_{G}$ of $G$.

If we fix $n$ and three partitions $\lambda, \mu$, and $\nu$ of $n$, we may consider the scalar products

$$
t_{\lambda, \mu, \nu}(q)=\left(\chi_{\lambda}^{q}, \chi_{\mu}^{q} \cdot \chi_{\nu}^{q}\right)_{\mathrm{GL}_{n}(q)} \quad \text { and } \quad \bar{t}_{\lambda, \mu, \nu}(q)=\left(\chi_{\lambda}^{q}, \chi_{\mu}^{q} \cdot \chi_{\nu}^{q}\right)_{\mathrm{GU}_{n}(q)}
$$

as functions in $q$. Using the terminology introduced above, we can now state the first result.

Proposition 2.1. (Mattig, [12, Propositon 3.1.6].) Let $n$ be a positive integer and let $\lambda, \mu, \nu$ be partitions of $n$. Then $t_{\lambda, \mu, \nu}(q)$ and $\bar{t}_{\lambda, \mu, \nu}(q)$, as functions on the set of all prime powers $q$, are polynomials in $q$ over $\mathbb{Q}$.

Let us sketch a proof of this result. First of all, the conjugacy classes of $G$ can be grouped together into class types, and the class types can be classified independently of $q$. Let us write $g_{s}$ and $g_{u}$ for the semisimple and unipotent part of an element $g \in G$, respectively. Two elements $g$ and $h$ of $G$ belong to the same class type, if $C_{G}\left(g_{s}\right)$ is conjugate to $C_{G}\left(h_{s}\right)$ and if $g_{u}$ is conjugate in $C_{G}\left(g_{s}\right)$ to a conjugate of $h_{u}$ lying in $C_{G}\left(g_{s}\right)$. (For a definition and more details on class types for general series of groups of Lie type see [6, Section 4].)

Secondly, the unipotent characters of $G$ are constant on class types, and thirdly, the numbers of conjugacy classes inside a class type, the lengths of conjugacy classes, and the values of the unipotent characters, viewed as functions of $q$, are polynomials in $q$ over the rationals. Finally, the order of $G$ is a polynomial in $q$ over the integers, and hence, by (1), the scalar products (2) are rational functions in $q$. Since these take integer values for all prime powers $q$, they are polynomials in $q$ over $\mathbb{Q}$.

Table 1 collects all the information that is needed to compute the tensor product polynomials for $n=2$. The first column of that table gives the number of conjugacy classes of $\mathrm{GL}_{2}(q)$ in each of the four class types, the second column gives the length of each conjugacy class in a class type. The third and fourth columns give the values of the two unipotent characters of $\mathrm{GL}_{2}(q)$.

We call such a table a generic unipotent character table. The CHEVIE-system [6] contains the generic unipotent character tables of $\mathrm{GL}_{n}(q)$ and $\mathrm{GU}_{n}(q)$ for $2 \leq n \leq$ 8, of $\mathrm{CSp}_{4}(q), \mathrm{CSp}_{6}(q), \mathrm{SO}_{8}^{-}(q)$, and $\operatorname{Spin}_{8}^{+}(q)$. Furthermore, CHEVIE includes programs to compute with these character tables. As examples we have computed 
TABLE 1. The generic unipotent character table of $\mathrm{GL}_{2}(q)$

\begin{tabular}{cccc}
\hline Nr. of Classes in Type & Length & $\chi_{(2)}$ & $\chi_{\left(1^{2}\right)}$ \\
\hline$q-1$ & 1 & 1 & $q$ \\
$q-1$ & $q^{2}-1$ & 1 & 0 \\
$\frac{1}{2}(q-1)(q-2)$ & $q(q+1)$ & 1 & 1 \\
$\frac{1}{2}(q-1) q$ & $q(q-1)$ & 1 & -1 \\
\hline
\end{tabular}

the polynomials $t_{\left(1^{5}\right),\left(1^{5}\right), \nu}(q)$ and $\bar{t}_{\left(1^{5}\right),\left(1^{5}\right), \nu}(q)$, where $\nu$ runs through the partitions of 5 . The result ist given in Table 2 .

Dirk Mattig has used CHEVIE to compute the polynomials $t_{\lambda, \mu, \nu}(q)$ for all triples of partitions of numbers up to 8 . Let us write $\mathbb{N}$ for the set of non-negative integers.

Observation 2.2. (Mattig, [12].) Let $\lambda, \mu, \nu$ be partitions of the integer $n$ with $1 \leq n \leq 8$. Then the function $t_{\lambda, \mu, \nu}(q)$ is a polynomial in $q$ over $\mathbb{N}$ for all prime powers $q$.

The same statement does not hold for the polynomials $\bar{t}_{\lambda, \mu, \nu}(q)$ but the calculations with CHEVIE lead to the following observation.

Observation 2.3. Let $\lambda, \mu, \nu$ be partitions of $n$, where $n$ is an integer with $1 \leq$ $n \leq 8$. Then we have:

(a) The functions $\bar{t}_{\lambda, \mu, \nu}(q)$ are polynomials in $q$ over the integers.

(b) The difference functions $t_{\lambda, \mu, \nu}(q)-\bar{t}_{\lambda, \mu, \nu}(q)$ are polynomials in $q$ over $2 \mathbb{N}$, that is the coefficients are even non-negative integers.

Remarks. There are formulae for the values of unipotent characters for $\operatorname{GL}_{n}(q)$ and $\mathrm{GU}_{n}(q)$ which are closely related by an operation called Ennola duality. Roughly speaking one has to substitute in the formulae for GL the parameter $q$ by $-q$ and to adjust some signs.

But this does not lead to a simple relation between $t_{\lambda, \mu, \nu}(q)$ and $\bar{t}_{\lambda, \mu, \nu}(q)$ (see the example in Table 2), since there is no such simple correspondence between the number of classes in class types (see the example in Table 3) and since the mentioned adjustment of signs depends of the class type.

Note also that the polynomials do not specialize to the corresponding scalar products of characters of the symmetric group $S_{n}$ at $q=1$. For example, if $n=2$ we have $(\mathrm{St}, \mathrm{St} \cdot \mathrm{St})_{\mathrm{GL}_{2}(q)}=1$. The Steinberg character of $\mathrm{GL}_{2}(q)$ corresponds to the alternating character of $S_{2}$, whose square equals the trivial character.

\section{Tensor Product Polynomials For Other Groups}

Now let $\{G(q) \mid q$ a prime power $\}$ be an arbritrary series of groups of Lie type. For example $\left\{\mathrm{Sp}_{4}(q)\right\}$, the symplectic groups on a 4 -dimensional vector space over $\mathbb{F}_{q}$, or $\left\{{ }^{2} E_{6}(q)_{s c}\right\}$ the twisted groups of type $E_{6}$ in a simply-connected algebraic group of this type. For a precise definition see [6, Section 4.1].

Again, each group $G(q)$ has a distinguished set of unipotent characters which are parametrized for all groups in the series independently of $q$ (see, e.g., [2, Sections $13.8,13.9]$ ). We write $\Lambda$ for a set of parameters of the unipotent characters and $\chi_{\lambda}^{q}$ for the character of $G(q)$ corresponding to $\lambda \in \Lambda$. The exact values of the 
TABLE 2. Some tensor products in $\mathrm{GL}_{5}(q)$ and in $\mathrm{GU}_{5}(q)$

\begin{tabular}{|c|c|c|}
\hline$\nu$ & $\left(\mathrm{St}_{G}, \mathrm{St}_{G} \cdot \chi_{\nu}\right)$ for $G=\mathrm{GL}_{5}(q)$ & $\left(\mathrm{St}_{G}, \mathrm{St}_{G} \cdot \chi_{\nu}\right)$ for $G=\mathrm{GU}_{5}(q)$ \\
\hline (5) & 1 & 1 \\
\hline$(4,1)$ & 4 & 0 \\
\hline$(3,2)$ & $q^{2}+2 q+5$ & $q^{2}+1$ \\
\hline$\left(3,1^{2}\right)$ & $q^{3}+2 q^{2}+4 q+6$ & $q^{3}+2 q+2$ \\
\hline$\left(2^{2}, 1\right)$ & $q^{4}+q^{3}+3 q^{2}+5 q+5$ & $q^{4}-q^{3}+q^{2}+q+1$ \\
\hline$\left(2,1^{3}\right)$ & $q^{5}+q^{4}+3 q^{3}+3 q^{2}+6 q+4$ & $q^{5}+q^{3}-q^{4}-q^{2}$ \\
\hline$\left(1^{5}\right)$ & $q^{6}+q^{4}+2 q^{3}+q^{2}+3 q+1$ & $q^{6}+q^{4}+q^{2}+q+1$ \\
\hline
\end{tabular}

TABLE 3. Numbers of conjugacy classes in class types

\begin{tabular}{cc}
\hline $\mathrm{GL}_{3}(q)$ & $\mathrm{GU}_{3}(q)$ \\
\hline$q-1$ & $q+1$ \\
$q-1$ & $q+1$ \\
$q-1$ & $q+1$ \\
$(q-1)(q-2)$ & $(q+1) q$ \\
$(q-1)(q-2)$ & $(q+1) q$ \\
$\frac{1}{6}(q-1)(q-2)(q-3)$ & $\frac{1}{6} q(q-1)(q+1)$ \\
$\frac{1}{2} q(q-1)^{2}$ & $\frac{1}{2}(q-2)(q+1)^{2}$ \\
$\frac{1}{3} q(q-1)(q+1)$ & $\frac{1}{3} q(q-1)(q+1)$ \\
\hline
\end{tabular}

unipotent characters are not yet known in all cases. But in those cases where they are known a certain generalization of Proposition 2.1 can be proved with the same arguments.

Unlike the case $\left\{\mathrm{GL}_{n}(q)\right\}$ it is in general no longer true that just one generic unipotent character table describes the values of the unipotent characters of $G(q)$ for all prime powers $q$. The smallest example for this is the series $\left\{\mathrm{SL}_{2}(q)\right\}$. For odd $q$ these groups have three unipotent conjugacy classes while for even $q$ there are only two.

It turns out that for each series $\{G(q)\}$ there is a number $m \in \mathbb{N}$ such that for any fixed $0 \leq i \leq m-1$ and all prime powers $q \equiv i(\bmod m)$ the class types can be parametrized independently of $q$ and the number of classes in a class type is given by a polynomial in $q$. Furthermore, the class length for all classes in a fixed class type is described by a polynomial in $q$.

It is not clear a priori that the unipotent characters are constant on class types. But this is true in all known cases. Moreover, in the known cases all values of unipotent characters are given by polynomials in $q$ with coefficients in some finite extension of $\mathbb{Q}$ which is independent of $q$. 
Thus, fixing an integer $0 \leq i \leq m-1$ such that the generic unipotent character table of $\{G(q) \mid q \equiv i(\bmod m)\}$ is known, we see as in Proposition 2.1 that the multiplicities

$$
t_{\lambda, \mu, \nu, i}(q)=\left(\chi_{\lambda}^{q}, \chi_{\mu}^{q} \cdot \chi_{\nu}^{q}\right)_{G(q)}
$$

for all prime powers $q$ with $q \equiv i(\bmod m)$ are polynomials in $q$ over $\mathbb{C}$.

If we compute these polynomials in the case $\left\{\operatorname{Sp}_{4}(q) \mid q\right.$ even $\}$ (the character values were first computed by Enomoto [5]), we quickly find polynomials with noninteger coefficients. This shows that Observations 2.2 and 2.3 do not generalize directly to other series of groups.

Almost Characters. To find such a generalization we have to look at the unipotent almost characters instead. These are certain $\mathbb{C}$-linear combinations, with coefficients independent of $q$, of the unipotent characters. Almost characters were introduced by Lusztig when he described the parametrizations of unipotent characters. The transforming matrices from irreducible characters to almost characters are called Fourier transform matrices.

For linear groups (like $\mathrm{GL}_{n}(q)$ and $\mathrm{SL}_{n}(q)$ ) the unipotent characters coincide with the unipotent almost characters. For unitary groups (like $\mathrm{GU}_{n}(q)$ and $\mathrm{SU}_{n}(q)$ ) they coincide up to sign. But for all other types of groups this is no longer true.

It is conjectured by Lusztig and proved in many cases by Lusztig and Shoji that the almost characters are class functions associated to certain geometric objects called character sheaves, see [10], [11] and [14].

Let $\tilde{\Lambda}$ be a set of parameters for the unipotent almost characters of the groups $G(q)$; for $\lambda \in \tilde{\Lambda}$ we write $\tilde{\chi}_{\lambda}^{q}$ for the corresponding almost character of $G(q)$. For $m$ as above and $0 \leq i \leq m-1$ set $\tilde{t}_{\lambda, \mu, \nu, i}(q)=\left(\tilde{\chi}_{\lambda}^{q}, \tilde{\chi}_{\mu}^{q} \cdot \tilde{\chi}_{\nu}^{q}\right)_{G(q)}$, viewed as a function on all prime powers $q \equiv i(\bmod m)$. Then, in those cases where we know that the $t_{\lambda, \mu, \nu, i}(q)$ are polynomials in $q$ the $\tilde{t}_{\lambda, \mu, \nu, i}(q)$ are also polynomials in $q$ over the complex numbers.

We have computed the polynomials $\tilde{t}_{\lambda, \mu, \nu, i}(q)$ for all $0 \leq i \leq m-1$ in the following cases. (Note, that the case $\mathrm{SL}_{n}(q)$ cannot easily be derived from the case $\mathrm{GL}_{n}(q)$ although the sets of unipotent characters are in bijection via restriction.)

\begin{tabular}{rc}
\hline \multicolumn{1}{c}{ Series } & $m$ \\
\hline$\left\{\mathrm{GL}_{n}(q)\right\}, n \leq 8$ & 1 \\
$\left\{\mathrm{SL}_{n}(q)\right\}, n \leq 8$ & $n$ \\
$\left\{\mathrm{GU}_{n}(q)\right\}, n \leq 8$ & 1 \\
$\left\{\mathrm{SU}_{n}(q)\right\}, n \leq 8$ & $n$ \\
$\left\{\mathrm{CO}_{n}(q)\right\}, n=7,9$ & 2 \\
\hline
\end{tabular}

\begin{tabular}{rr}
\hline Series & $m$ \\
\hline$\left\{\operatorname{CSp}_{n}(q)\right\}, n=4,6,8$ & 2 \\
$\left\{\operatorname{Sp}_{n}(q)\right\}, n=4,6,8$ & 2 \\
$\left\{\operatorname{Spin}_{7}(q)\right\}$ & 4 \\
$\left\{\operatorname{Spin}_{8}^{+}(q)\right\}$ & 2 \\
$\left\{{ }^{3} D_{4}(q)\right\}$ & 2 \\
\hline
\end{tabular}

Some of these tables of unipotent characters were available in CHEVIE and computed in [16], [5], [13], [4], [7], and [9]. The other non-linear group cases were computed by the second author using the results from [15].

Observation 3.1. In all cases mentioned above, the functions $\tilde{t}_{\lambda, \mu, \nu, i}(q)$ are polynomials in $q$ over $\mathbb{Z}$. Here, some negative coefficients occur in the cases $\mathrm{GU}_{n}(q)$ with $5 \leq n \leq 8, \operatorname{SU}_{n}(q)$ with $3 \leq n \leq 8$ (for some $i$ ), ${ }^{3} D_{4}(q)$ and $\operatorname{Spin}_{7}(q)$ with $q \equiv 3(\bmod 4)$. 
This suggests that, if our observations are actually true for all series of groups of Lie type, one has to consider character sheaves for an explanation. Comparing the cases GL and GU it is interesting to know that the character sheaves leading to the values of unipotent characters are the same geometric objects for GL and GU. Maybe there is an interpretation of the coefficients of the tensor product polynomials using the same non-negative numbers which have to be added up with different signs.

There does not seem to be an obvious notion of tensor products of character sheaves which correspond to the tensor products of the associated class functions.

\section{Special Results on the tensor PRODUCT POLYNOMials}

When the first author gave the talk on these results in Gainesville, Klaus Lux and Gunter Malle observed that the constant coefficient of the polynomial $t_{\left(1^{5}\right),\left(1^{5}\right), \nu}$ in the first half of Table 2 equals $\zeta^{\nu}(1)$, where $\zeta^{\nu}$ is the irreducible character of the symmetric group $S_{5}$ labelled by the partition $\nu$. Gunter Malle observed furthermore, that the constant coefficient of the polynomial $\bar{t}_{\left(1^{5}\right),\left(1^{5}\right), \nu}$ in the second half of Table 2 equals 0 , if $\chi_{\nu}$ does not lie in the principal series, and equals the degree of the corresponding character of the Weyl group of $\mathrm{GU}_{5}(q)$, otherwise. Here, the Weyl group is a dihedral group of order 8 .

During the conference a sketch of a proof for these facts emerged through discussions with Gunter Malle and Meinolf Geck. We give the details here.

Proposition 4.1. Let $n$ be a positive integer and let $\nu$ be a partition of $n$. Write $\zeta^{\nu}$ for the irreducible character of the symmetric group $S_{n}$ labelled by $\nu$. Put $m:=$ $\lfloor n / 2\rfloor$.

(a) The constant coefficient of $t_{\left(1^{n}\right),\left(1^{n}\right), \nu}$ equals $\zeta^{\nu}(1)$.

(b) The constant coefficient of $\bar{t}_{\left(1^{n}\right),\left(1^{n}\right), \nu}$ equals $\left|\zeta^{\nu}(\sigma)\right|$, where $\sigma \in S_{n}$ is an involution that is a product of $m$ disjoint transpositions.

The value $\left|\zeta^{\nu}(\sigma)\right|$ also has the following interpretation. Let $\kappa$ and $(\alpha, \beta)$ denote the 2-core and the 2-quotient of $\nu$, respectively. Then $\left|\zeta^{\nu}(\sigma)\right|$ equals 0 , if $|\kappa|>1$, and $\zeta^{(\alpha, \beta)}(1)$, otherwise. Here, $\zeta^{(\alpha, \beta)}$ denotes the irreducible character of the Weyl group of type $B_{m}$ labelled by the bi-partition $(\alpha, \beta)$ of $m$.

Proof. For $0 \neq f \in \mathbb{Q}[X], f=X^{l} g$ with $l \geq 0$ and $X \nmid g$, we write $f_{X}:=X^{l}$ and $f_{X^{\prime}}:=g=f / f_{X}$.

The proof is given simultaneously for the general linear and unitary groups. Let $\varepsilon$ be a parameter taking the values \pm 1 . Let $\mathbb{G}_{\varepsilon}$ denote the generic group giving rise to the series $\operatorname{GL}_{n}(q)$, if $\varepsilon=1$, and to $\mathrm{GU}_{n}(q)$, if $\varepsilon=-1$. We write $\left|\mathbb{G}_{\varepsilon}\right|$ for the order polynomial of $\mathbb{G}_{\varepsilon}$, i.e., the value of $\left|\mathbb{G}_{\varepsilon}\right|$ at $q$ is the order of the finite group $G_{\varepsilon}:=\mathbb{G}_{\varepsilon}(q)$. Letting $N:=n(n-1) / 2$, we have

$$
\left|\mathbb{G}_{\varepsilon}\right|=X^{N} \prod_{i=1}^{n}\left(X^{i}-\varepsilon^{i}\right) .
$$

Let $\tilde{\Omega}$ be a set of labels for the class types of $\mathbb{G}_{\varepsilon}$ and let $\Omega \subseteq \tilde{\Omega}$ label the class types of semisimple elements. Such sets can be chosen independently of $\varepsilon$. For $\omega \in \tilde{\Omega}$ we write $\operatorname{St}_{\varepsilon}(\omega)$ and $\chi_{\nu, \varepsilon}(\omega)$ for the value polynomials of the Steinberg character and the unipotent character $\chi_{\nu}$, respectively. We also write $n_{\varepsilon}(\omega)$ and $c_{\varepsilon}(\omega)$ for the numbers of conjugacy classes of class type $\omega$ and the numbers of elements of a conjugacy class of class type $\omega$, respectively. Finally, $t^{\varepsilon}$ denotes $t$, if $\varepsilon=1$, and $\bar{t}$, 
if $\varepsilon=-1$. We then have

$$
t_{\left(1^{n}\right),\left(1^{n}\right), \nu}\left|\mathbb{G}_{\varepsilon}\right|=\sum_{\omega \in \tilde{\Omega}} n_{\varepsilon}(\omega) c_{\varepsilon}(\omega) \operatorname{St}_{\varepsilon}(\omega)^{2} \chi_{\nu, \varepsilon}(\omega) .
$$

This follows from Formula (1) with the above settings and the fact that $\chi_{\nu, \varepsilon}(\omega)=$ $\chi_{\nu, \varepsilon}\left(\omega^{\prime}\right)$, where $\omega^{\prime}$ denotes the class type containing the inverses of the elements of class type $\omega$.

If $\omega \in \tilde{\Omega} \backslash \Omega$, then $\operatorname{St}_{\varepsilon}(\omega)=0$, and if $\omega \in \Omega$, then $\operatorname{St}_{\varepsilon}(\omega)= \pm\left(\left|\mathbb{G}_{\varepsilon}\right| / c_{\varepsilon}(\omega)\right)_{X}$ (see $\left[2\right.$, Theorem 6.5.9]). Note that $c_{\varepsilon}(\omega)$ divides $\left|\mathbb{G}_{\varepsilon}\right|$, and that $\left|\mathbb{G}_{\varepsilon}\right| / c_{\varepsilon}(\omega)$ is the centralizer order polynomial corresponding to the class type $\omega$. Let us write $\left(\left|\mathbb{G}_{\varepsilon}\right| / c_{\varepsilon}(\omega)\right)_{X}=: X^{m_{\omega, \varepsilon}}$. Then, dividing both sides of the equation above by $X^{N}$, we have

$$
t_{\left(1^{n}\right),\left(1^{n}\right), \nu}^{\varepsilon}\left|\mathbb{G}_{\varepsilon}\right|_{X^{\prime}}=\sum_{\omega \in \Omega} n_{\varepsilon}(\omega) c_{\varepsilon}(\omega)_{X^{\prime}} X^{m_{\omega, \varepsilon}} \chi_{\nu, \varepsilon}(\omega) .
$$

Now $m_{\omega, \varepsilon}=0$ for $\omega \in \Omega$, if and only if $\omega$ is a class type of regular semisimple elements, i.e., the generic centralizer is a torus. So, only regular semisimple classes contribute to the constant term of the polynomial $t_{\left(1^{n}\right),\left(1^{n}\right), \nu}^{\varepsilon}$.

Finally, we have to consider the polynomials $n_{\varepsilon}(\omega)$ for class types of regular semisimple elements. Such class types are parametrized by the partitions of $n$ as follows. Given a regular semisimple element in $G_{\varepsilon}$, it has $n$ pairwise different eigenvalues and the set of eigenvalues is permuted by raising these numbers to the $\varepsilon q$-th power. All such sets of eigenvalues occur and each set of eigenvalues occurs for exactly one conjugacy class. The class type of the element is parametrized by the cycle type of this permutation on the eigenvalues.

Using induction one can see that the number of sets of eigenvalues as above for a fixed cycle type is a polynomial in $q$ which is divisible by $q$ if there is:

- any cycle of length greater than one, if $\varepsilon=1$.

- more than one cycle of length one or a cycle of length greater than two, if $\varepsilon=-1$.

This shows that in each case there is only one class type, namely the one of the regular elements in a maximally split torus, which contributes to the constant term we are interested in. Let $\omega_{0}$ be the label of this class type. Then

$$
t_{\left(1^{n}\right),\left(1^{n}\right), \nu}^{\varepsilon}\left|\mathbb{G}_{\varepsilon}\right|_{X^{\prime}} \equiv n_{\varepsilon}\left(\omega_{0}\right) c_{\varepsilon}\left(\omega_{0}\right)_{X^{\prime}} \chi_{\nu, \varepsilon}\left(\omega_{0}\right)(\bmod X) .
$$

Now, for $\varepsilon=1$, i.e., for $\mathrm{GL}_{n}(q)$, we find from the combinatorial interpretation of $n_{1}\left(\omega_{0}\right)$ given above that

$$
n_{1}\left(\omega_{0}\right)=\frac{1}{n !} \prod_{i=1}^{n}(X-i)
$$

Since

$$
c_{1}\left(\omega_{0}\right)_{X^{\prime}}=\frac{\prod_{i=1}^{n}\left(X^{i}-1\right)}{(X-1)^{n}}=\frac{\left|\mathbb{G}_{\varepsilon}\right|_{X^{\prime}}}{(X-1)^{n}},
$$

it follows that the constant coefficient of $t_{\left(1^{n}\right),\left(1^{n}\right), \nu}$ equals $\chi_{\nu, 1}\left(\omega_{0}\right)$.

Now let $\varepsilon=-1$. In this case we have

$$
n_{-1}\left(\omega_{0}\right)=\frac{1}{2^{m} m !}(X+1)^{n-2 m} \prod_{i=1}^{m}\left(X^{2}-X-2 i\right),
$$


and

$$
c_{-1}\left(\omega_{0}\right)_{X^{\prime}}=\frac{\prod_{i=1}^{n}\left(X^{i}-(-1)^{i}\right)}{(X+1)^{n-2 m} \prod_{i=1}^{m}\left(X^{2}-1\right)} .
$$

It follows that the constant coefficient of $\bar{t}_{\left(1^{n}\right),\left(1^{n}\right), \nu}$ equals $\chi_{\nu,-1}\left(\omega_{0}\right)$.

To determine the value of $\chi_{\nu, \varepsilon}\left(\omega_{0}\right)$ we use the character formula for the values of Deligne-Lusztig generalized characters on semisimple elements (see [2, Proposition 7.5.3]), and the connection of unipotent characters with unipotent almost characters as presented in $[1$, p. 45$]$.

Let $\psi_{\nu, \varepsilon}$ denote the unipotent almost character of $G_{\varepsilon}$ corresponding to $\zeta^{\nu}$. Then $\psi_{\nu, \varepsilon}=\chi_{\nu, \varepsilon}$, if $G_{\varepsilon}=\mathrm{GL}_{n}(q)$, and $\psi_{\nu, \varepsilon}= \pm \chi_{\nu, \varepsilon}$, otherwise. Moreover, $\psi_{\nu, 1}\left(\omega_{0}\right)=$ $\zeta^{\nu}(1)$, so we are done in case $\varepsilon=1$. Let $\varepsilon=-1$. Then $\psi_{\nu,-1}\left(\omega_{0}\right)=\zeta^{\nu}(\sigma)$. Also, by [8, Corollary 2.7.33], $\left|\zeta^{\nu}(\sigma)\right|=0$, if $|\kappa|>1$, and $\left|\zeta^{\nu}(\sigma)\right|=\zeta^{(\alpha, \beta)}(1)$, if $|\kappa| \leq 1$. It remains to show that the sign of $\zeta^{\nu}(\sigma)$ equals the sign of $\psi_{\nu,-1}$. By the definition of the almost characters, the degree of $\psi_{\nu,-1}$ is obtained from the degree of $\psi_{\nu, 1}$ by replacing $q$ by $-q$. Thus $\psi_{\nu,-1}=\chi_{\nu,-1}$, if the generic degree polynomial has even degree, and $\psi_{\nu,-1}=-\chi_{\nu,-1}$, otherwise. A formula for the generic degree polynomial can be found in $\left[2, \mathrm{p}\right.$. 466]. The sign of $\zeta^{\nu}(\sigma)$ is determined in [8, pp. 80-82]. Using these descriptions and induction on the 2-weight of $\nu$, it follows that $\psi_{\nu,-1}=-\chi_{\nu,-1}$ if and only if $\zeta^{\nu}(\sigma)$ is negative.

Remark. Note that the property that all but one of the numbers of classes in the class types of regular semisimple elements is divisible by $q$ is a special property of the series $\mathrm{GL}_{n}(q)$ and $\mathrm{GU}_{n}(q)$. Thus there is no generalization of Theorem 4.1 to other series of groups.

Let us now fix the positive integer $n$ and put $G=\mathrm{GL}_{n}(q)$ for the remainder of this section. The following result is also contained in Mattig's PhD-thesis.

Proposition 4.2. (Mattig, [12, Theorem 6.4.8].) For all partitions $\mu, \nu$ of $n$, the polynomial $t_{(n-1,1), \mu, \nu}$ is constant (and hence a non-negative integer).

In order to prove this, Mattig considers the permutation characters $1_{P_{\lambda}}{ }^{G}$ on parabolic subgroups $P_{\lambda}$. Here, $\lambda$ again is a partition of $n$ and $P_{\lambda}$ denotes the corresponding parabolic subgroup of $G$ (whose Levi subgroup is the group of block diagonal matrices with blocks of sizes given by the parts of $\lambda$ ).

Let us write $\mathcal{P}_{n}$ for the set of partitions of $n$. Given $\lambda \in \mathcal{P}_{n}$, there are nonnegative integers $a_{\lambda, \mu}$, independent of $q$, such that

$$
1_{P_{\lambda}}{ }^{G}=\sum_{\mu \in \mathcal{P}_{n}} a_{\lambda, \mu} \chi_{\mu}^{q}
$$

(see, e.g., [3, Theorem (70.24)]). Moreover, by [8, Section 2.2], the matrix of coefficients $\left(a_{\lambda, \mu}\right)_{\lambda, \mu \in \mathcal{P}_{n}}$ is invertible over the integers. In particular, the unipotent characters $\chi_{\lambda}^{q}$ can be expressed as $\mathbb{Z}$-linear combinations of the permutation characters $1_{P_{\mu}}{ }^{G}$. For example, $\chi_{(n-1,1)}^{q}=1_{P_{(n-1,1)}}{ }^{G}-1_{P_{(n)}}{ }^{G}=1_{P_{(n-1,1)}}{ }^{G}-1_{G}$.

It follows from Proposition 2.1 and Equation (3) that there are polynomials $r_{\lambda, \mu, \nu} \in \mathbb{Q}[X]$ such that

$$
r_{\lambda, \mu, \nu}(q)=\left(1_{P_{\lambda}}^{G}, 1_{P_{\mu}}^{G} \cdot 1_{P_{\nu}}^{G}\right)_{\mathrm{GL}_{n}(q)}
$$


for all prime powers $q$. Another interpretation of these polynomials is provided by

$$
\left(1_{P_{\lambda}}{ }^{G}, 1_{P_{\mu}}{ }^{G} \cdot 1_{P_{\nu}}{ }^{G}\right)_{\mathrm{GL}_{n}(q)}=\sum_{x \in D_{\mu, \nu}}\left|P_{\lambda} \backslash G / P_{\mu} \cap{ }^{x} P_{\nu}\right|
$$

where $D_{\mu, \nu}$ denotes the set of distinguished double coset representatives corresponding to the parabolic subgroups $P_{\mu}$ and $P_{\nu}$. Note that $D_{\mu, \nu}$ can be chosen, independently of $q$, as a subset of distinguished double coset representatives of the Weyl group $S_{n}$ of $G$. Using (4), Mattig shows that $r_{(n-1,1), \mu, \nu}$ is constant for all partitions $\mu, \nu$ of $n$. The remarks following (3) now prove Proposition 4.2.

Of course, $r_{\lambda, \mu, \nu} \in \mathbb{N}[X]$ if $t_{\lambda, \mu, \nu} \in \mathbb{N}[X]$.

Let us consider a further special case, namely $P_{\left(1^{n}\right)}=B$. We have

$$
1_{B}{ }^{G}=\sum_{\lambda \in \mathcal{P}_{n}} \zeta^{\lambda}(1) \chi_{\lambda}^{q}
$$

Moreover, by [2, Propositions 7.4.4 and 7.5.4] we have

$$
1_{B}{ }^{G} \cdot \mathrm{St}_{G}=1_{T}^{G}
$$

where $T$ denotes the maximally split torus of $G$ consisting of the diagonal matrices. Using Proposition 2.1, and Equation (5), we see that there is a polynomial $s_{n} \in$ $\mathbb{Q}[X]$ such that

$$
\begin{aligned}
s_{n}(q) & =|B \backslash G / T| \\
& =\left(1_{B}{ }^{G}, 1_{T}{ }^{G}\right)_{G} \\
& =\left(1_{B}{ }^{G}, 1_{B}{ }^{G} \cdot \mathrm{St}_{G}\right)_{G} \\
& =\sum_{\lambda, \mu \in \mathcal{P}_{n}} \zeta^{\lambda}(1) \zeta^{\mu}(1) t_{\lambda, \mu,\left(1^{n}\right)}(q) .
\end{aligned}
$$

Theorem 4.3. (Mattig, [12, Chapters 4 and 8].) The polynomials $s_{n}$ have integer coefficients for all $n \geq 1$. The degree of $s_{n}$ equals $(n-1)(n-2) / 2$, and the coefficient at $X^{i}$ is positive for all $0 \leq i \leq(n-1)(n-2) / 2$. Moreover, $s_{n}$ is monic except for $n=2$.

As examples, we give the polynomials $s_{n}$ for $1 \leq n \leq 6$.

$$
\begin{aligned}
s_{1}= & 1 \\
s_{2}= & 3 \\
s_{3}= & X+19 \\
s_{4}= & X^{3}+6 X^{2}+36 X+211 \\
s_{5}= & X^{6}+8 X^{5}+35 X^{4}+136 X^{3}+ \\
& 410 X^{2}+1253 X+3651 \\
s_{6}= & X^{10}+10 X^{9}+54 X^{8}+209 X^{7}+ \\
& 685 X^{6}+1969 X^{5}+4951 X^{4}+11592 X^{3}+ \\
& 24415 X^{2}+50547 X+90921
\end{aligned}
$$

Let us sketch the main arguments in the proof of Theorem 4.3. Let $U$ denote the set of upper unitriangular matrices of $G$ so that $B=U T$. Let $W$ denote the Weyl group of $G$ and $w_{0}$ its longest element. For any $w \in W$, put $U_{w}:=U \cap U^{w_{0} w}$. Then $U_{w}$ is invariant under conjugation by $T$, and $R:=\left\{w u \mid w \in W, u \in U_{w}\right\}$ is a set of coset representatives of the right coset of $B$ in $G$. Moreover, for $w u, w^{\prime} u^{\prime} \in R$, 
we have $B w u T=B w^{\prime} u^{\prime} T$ if and only if $w=w^{\prime}$ and $u$ and $u^{\prime}$ are in the same orbit of $T$ on $U_{w}$, where $T$ acts on $U_{w}$ by conjugation.

This shows that it suffices to determine, for each $w \in W$, the number of orbits of $T$ on $U_{w}$ as a function of $q$. Fix $w \in W$ and write $V:=U_{w}$. Let $\Delta_{+}$denote the set of pairs $\{(i, j) \mid 1 \leq i<j \leq n\}$, which corresponds to the set of positive roots of $G$. Then there is a subset $\Xi \subseteq \Delta_{+}$such that

$$
V=\left\{\left(a_{i j}\right) \in \mathbb{F}_{q}^{n \times n} \mid a_{i i}=1 \text { for } 1 \leq i \leq n, \text { and } a_{i j}=0 \text { for } i \neq j \text { and }(i, j) \notin \Xi\right\}
$$

(see [2, Proposition 2.5.16]).

Choose an injective mapping $\nu: \Xi \rightarrow \mathbb{N}$. A subset $\Lambda \subseteq \Xi$ can then be viewed as a weighted undirected graph, by associating $\nu(i, j)$ to the edge $(i, j) \in \Lambda$. Since the weight function is injective, $\Lambda$ has a unique minimal spanning forest. Let $\mathcal{X}$ denote the set of subsets of $\Xi$ which arise as minimal spanning forests in this way. Then $\mathcal{X}$ contains a unique maximal element $\Gamma_{\max }$.

For a subset $\Lambda$ of $\Xi$ let

$$
V_{\Lambda}^{*}:=\left\{\left(a_{i j}\right) \in V \mid a_{i j} \neq 0 \text { if and only if } i=j \text { or }(i, j) \in \Lambda\right\} .
$$

For $\Gamma \in \mathcal{X}$, let $V_{\Gamma}$ denote the union of the sets $V_{\Lambda}^{*}$, where $\Lambda$ runs through the subsets of $\Xi$ which have $\Gamma$ as their minimal spanning forest. Then, obviously, $V_{\Gamma}$ is invariant under conjugation by $T$ (since every $V_{\Lambda}^{*}$ is $T$-invariant), and

$$
V=\bigcup_{\Gamma \in \mathcal{X}} V_{\Gamma}
$$

a disjoint union.

The proof concludes by showing that the number of $T$-orbits on $V_{\Gamma}$ equals $q^{n_{\Gamma}}$, where $n_{\Gamma}=\left|\Gamma_{\max }\right|-|\Gamma|$.

\section{REFERENCES}

[1] M. Broué, G. Malle, and J. Michel, Generic blocks of finite reductive groups, in: Représentations unipotentes génériques et blocs des groupes réductifs finis, Astérisque 212 (1993), 7-92.

[2] R. W. Carter, Finite Groups of Lie Type: Conjugacy Classes and Complex Characters, Wiley, 1985.

[3] C. W. Curtis and I. Reiner, Methods of Representation Theory I, Wiley, 1981; II, Wiley, 1987.

[4] D. I. Deriziotis and G. O. Michler, Character tables and blocks of finite simple triality groups ${ }^{3} D_{4}\left(q^{3}\right)$, Trans. Amer. Math. Soc. 303 (1987), 39-70.

[5] H.Enомото, The characters of the finite symplectic group $\operatorname{Sp}(4, q), q=2^{f}$, Osaka J. Math. 9 (1972), 75-94.

[6] M. Geck, G. Hiss, F. Lübeck, G. Malle, and G. Pfeiffer, ChEVIE-A system for computing and processing generic character tables, AAECC 7 (1996), 175-210.

[7] M. GeCK AND G. Pfeiffer, Unipotent characters of the Chevalley groups $D_{4}(q), q$ odd, Manuscripta Math. 76 (1992), 281-304.

[8] G. D. James And A. Kerber, The Representation Theory of the Symmetric Group, Encyclopedia Math. 16, 1988.

[9] F. LüBECK, Charaktertafeln für die Gruppen $\operatorname{CSp}_{6}(q)$ mit ungeradem $q$ und $\operatorname{Sp}_{6}(q)$ mit geradem $q$, Dissertation, Universität Heidelberg, 1993.

[10] G. Lusztig, Character sheaves I to V, Adv. in Math. 56, 57, 59, 61, 1985/1986.

[11] G. Lusztig, Green functions and character sheaves, Ann. Math. 131 (1990), 355-408.

[12] D. Mattig, Tensor Products of Unipotent Characters of $G L(n, q)$, Dissertation, Universität Heidelberg, 1999.

[13] K. ShinodA, The characters of the finite conformal symplectic group $\operatorname{CSp}(4, q)$, Comm. Algebra 10 (1982), 1369-1419. 
[14] T. ShoJI, Character sheaves and almost characters of reductive groups I and II, Adv. in Math. 111 (1995), 244-313, 314-354.

[15] T. SнолI, Unipotent characters of finite classical groups, in: Finite reductive groups (Luminy, 1994), Birkhäuser, 1997, 373-413.

[16] B. SRInivasan, The characters of the finite symplectic group $\operatorname{Sp}(4, q)$, Trans. Amer. Math. Soc. 131 (1968), 488-525.

Lehrstuhl D für Mathematik, RWTH Aachen, 52056 Aachen, Germany

E-mail address: G.H.: gerhard.hiss@math.rwth-aachen.de

E-mail address: F.L.: frank.luebeck@math.rwth-aachen.de 